\title{
Quantification of central fatigue: a central debate
}

\author{
Nicolas Place ${ }^{1}[$ \\ Received: 27 April 2021 / Accepted: 5 May 2021 / Published online: 15 May 2021 \\ (c) The Author(s), under exclusive licence to Springer-Verlag GmbH Germany, part of Springer Nature 2021
}

The transient impairment in neuromuscular function in response to intense or prolonged exercise, namely muscle fatigue, has fascinated scientists for more than a century. Despite the fact that several thousands of papers have been published, there is still no consensus in the literature on many aspects related to muscle fatigue, including the definition/taxonomy (Kluger et al. 2013; Place and Millet 2020). Despite this ongoing debate, there is a general consensus around the idea that an acute reduction in the force generating capacity is caused by alterations located above and/ or beyond the neuromuscular junction, i.e., central (neural) and/or intramuscular (peripheral) processes, respectively. It is reasonably well accepted that peripheral fatigue in humans is assessed through the change in the amplitude of an evoked force while the stimulated muscle is a rest, although factors such as the competition between fatigue and potentiation and the delay between the end of the exercise and the actual quantification may bias the extent of peripheral fatigue. Any exercise-induced reduction in the amplitude of the force in response to a single stimulation (muscle twitch), paired (doublet) or more (tetanic contraction) stimuli is considered as an evidence of impairment between the site of stimulation (usually the motor nerve) to the interaction of contractile proteins. These steps may include failure of action potential generation/propagation, excitation-contraction coupling, $\mathrm{Ca}^{2+}$ release from the sarcoplasmic reticulum, myofibrillar $\mathrm{Ca}^{2+}$ sensitivity and/or force produced at the cross-bridge level.

From a conceptual viewpoint, it is more difficult to quantify central fatigue, i.e., the potential impairment originating from the central nervous system. The seminal work of Merton (1954) is considered as an important step forward in the field as it proposes a method to quantify a potential neural

\section{Communicated by Michael Lindinger .}

Nicolas Place

nicolas.place@unil.ch

1 Institute of Sport Sciences, University of Lausanne, Lausanne, Switzerland defect by measuring the force in response to an electrical stimulation superimposed to a voluntary contraction. This technique is known as the twitch interpolation technique (or the interpolated twitch technique) and is commonly accepted to be the gold standard of central fatigue measurement. In his influential review, Gandevia (2001) defines central fatigue as a 'progressive reduction in voluntary activation of muscle during exercise'. Thus, voluntary activation, which is usually quantified using the twitch interpolation technique, and central fatigue are intimately linked. Any exercise-induced reduction in the voluntary activation level is usually interpreted as an evidence of central fatigue. Occasionally, there have been reports suggesting that caution should be taken when quantifying voluntary activation with the twitch interpolation technique, which may overestimate (1) the ability to maximally drive the active muscles (Kooistra et al. 2007) or (2) the extent of central fatigue (Place et al. 2008). This led to an open debate 12 years ago (De Haan et al. 2009; Taylor 2009) that is still ongoing (Gandevia et al. 2013; Cheng et al. 2013; Neyroud et al. 2016; Contessa et al. 2016). In their review published in this issue of the European Journal of Applied Physiology, Dotan et al. (2021) summarize evidence questioning the validity and reliability of the twitch interpolation technique to quantify central fatigue.

There is clear evidence from the literature that intramuscular factors cannot solely explain the fatigue process (e.g., placebo effect; mental fatigue; 'burst' of short, intense muscle activity at task failure, etc.), i.e., central fatigue does exist. However, the ongoing debate surrounds the quantification of central fatigue. The twitch interpolation technique offers the advantage to be easily quantified but it is mostly limited to maximal isometric contraction and the relevance of the reduction in voluntary activation to exercise performance during submaximal intensity tasks is unclear (Brownstein et al. 2021). In their review, Dotan et al. (2021) advance several arguments suggesting that the variables used for the calculation of voluntary activation are affected by peripheral factors or factors independent of muscle fatigue, questioning the use of the twitch interpolation technique to determine central fatigue. 
A pragmatic suggestion would thus be to quantify other variables together with voluntary activation to better apprehend neural adaptations to exercise. For instance, Trajano et al. (2013) combined measurements of voluntary activation with electromyographic activity and $\mathrm{V}$ wave obtained during maximal efforts and normalized by the compound muscle action potential (M-wave) amplitude to better quantify changes in central drive after a passive stretch intervention. When performing a submaximal exercise at a constant intensity, quantification of the increase in the rate of perceived exertion might also be valuable (Taylor and Gandevia 2008). Similarly, changes in motor unit firing and force can be characterized simultaneously to assess central fatigue during submaximal contractions (Taylor and Gandevia 2008). Finally, the rate of force development measured in the early phase of an explosive contraction (first 50-75 ms) is thought to depend mainly on motor unit firing rate and as such may be used as a surrogate for neural function (Maffiuletti et al. 2016). In this context, the recent development of high-density surface electromyography (Del Vecchio et al. 2019,2020 ) will be helpful for a more precise characterization of central fatigue in the near future.

In conclusion, the arguments presented here by Dotan et al. (2021) should be considered by scientists, who should be aware of the limitations of the technique. Using multiple indicators of central fatigue is advised to obtain a more comprehensive view of the complex neural adaptations occurring during exercise and recovery.

\section{Author contributions NP wrote the Editorial.}

\section{Declarations}

Conflict of interest The author declares no conflict of interest.

\section{References}

Brownstein CG, Millet GY, Thomas K (2021) Neuromuscular responses to fatiguing locomotor exercise. Acta Physiol (Oxf) 231(2):e13533. https://doi.org/10.1111/apha.13533

Cheng AJ, Dalton BH, Harwood B, Power GA (2013) "SIT" down and relax: the interpolated twitch technique is still a valid measure of central fatigue during sustained contraction tasks. J Physiol 591(15):3677-3678. https://doi.org/10.1113/jphysiol.2013. 258566

Contessa P, Puleo A, De Luca CJ (2016) Is the notion of central fatigue based on a solid foundation? J Neurophysiol 115(2):967-977. https://doi.org/10.1152/jn.00889.2015

de Haan A, Gerrits KH, de Ruiter CJ (2009) Counterpoint: the interpolated twitch does not provide a valid measure of the voluntary activation of muscle. J Appl Physiol 107(1):355-357. https://doi. org/10.1152/japplphysiol.91220.2008a
Del Vecchio A, Casolo A, Negro F, Scorcelletti M, Bazzucchi I, Enoka R, Felici F, Farina D (2019) The increase in muscle force after 4 weeks of strength training is mediated by adaptations in motor unit recruitment and rate coding. J Physiol 597(7):1873-1887. https://doi.org/10.1113/JP277250

Del Vecchio A, Holobar A, Falla D, Felici F, Enoka RM, Farina D (2020) Tutorial: analysis of motor unit discharge characteristics from high-density surface EMG signals. J Electromyogr Kinesiol 53:102426. https://doi.org/10.1016/j.jelekin.2020.102426

Dotan R, Woods S, Contessa P (2021) On the reliability and validity of central fatigue determination. Eur J Appl Physiol. https://doi. org/10.1007/s00421-021-04700-w

Gandevia SC (2001) Spinal and supraspinal factors in human muscle fatigue. Physiol Rev 81(4):1725-1789. https://doi.org/10.1152/ physrev.2001.81.4.1725

Gandevia SC, McNeil CJ, Carroll TJ, Taylor JL (2013) Twitch interpolation: superimposed twitches decline progressively during a tetanic contraction of human adductor pollicis. J Physiol 591(5):1373-1383. https://doi.org/10.1113/jphysiol.2012.248989

Kluger BM, Krupp LB, Enoka RM (2013) Fatigue and fatigability in neurologic illnesses: proposal for a unified taxonomy. Neurology 80(4):409-416. https://doi.org/10.1212/WNL.0b013e31827f07be

Kooistra RD, de Ruiter CJ, de Haan A (2007) Conventionally assessed voluntary activation does not represent relative voluntary torque production. Eur J Appl Physiol 100(3):309-320. https://doi.org/ 10.1007/s00421-007-0425-8

Maffiuletti NA, Aagaard P, Blazevich AJ, Folland J, Tillin N, Duchateau J (2016) Rate of force development: physiological and methodological considerations. Eur J Appl Physiol 116(6):1091-1116. https://doi.org/10.1007/s00421-016-3346-6

Merton PA (1954) Voluntary strength and fatigue. J Physiol 123(3):553-564. https://doi.org/10.1113/jphysiol.1954.sp005070

Neyroud D, Cheng AJ, Bourdillon N, Kayser B, Place N, Westerblad H (2016) Muscle Fatigue affects the interpolated twitch technique when assessed using electrically-induced contractions in human and rat muscles. Front Physiol 7:252. https://doi.org/10.3389/ fphys.2016.00252

Place N, Millet GY (2020) Quantification of neuromuscular fatigue: what do we do wrong and why? Sports Med 50(3):439-447. https://doi.org/10.1007/s40279-019-01203-9

Place N, Yamada T, Bruton JD, Westerblad H (2008) Interpolated twitches in fatiguing single mouse muscle fibres: implications for the assessment of central fatigue. J Physiol 586(11):2799-2805. https://doi.org/10.1113/jphysiol.2008.151910

Taylor JL (2009) Point: the interpolated twitch does/does not provide a valid measure of the voluntary activation of muscle. J Appl Physiol 107(1):354-355. https://doi.org/10.1152/japplphysiol. 91220.2008

Taylor JL, Gandevia SC (2008) A comparison of central aspects of fatigue in submaximal and maximal voluntary contractions. $\mathbf{J}$ Appl Physiol 104(2):542-550. https://doi.org/10.1152/japplphysi ol.01053.2007

Trajano GS, Seitz L, Nosaka K, Blazevich AJ (2013) Contribution of central vs. peripheral factors to the force loss induced by passive stretch of the human plantar flexors. J Appl Physiol 115(2):212218. https://doi.org/10.1152/japplphysiol.00333.2013

Publisher's Note Springer Nature remains neutral with regard to jurisdictional claims in published maps and institutional affiliations. 